Title

\title{
Comparative Connectomics
}

\section{Authors}

Martijn P. van den Heuvel ${ }^{1}$, Edward T. Bullmore ${ }^{2,3}$, Olaf Sporns ${ }^{4,5}$

\section{Affiliations}

1. Department of Psychiatry, Brain Center Rudolf Magnus, University Medical Center Utrecht, Utrecht, The Netherlands

2. Department of Psychiatry, University of Cambridge, Cambridge, UK

3. ImmunoPsychiatry, Academic Discovery Performance Unit, GlaxoSmithKline, Cambridge, UK

4. Department of Psychological and Brain Sciences, Indiana University, Bloomington, USA

5. Indiana University Network Science Institute, Indiana University, Bloomington, USA

\section{Corresponding author}

Martijn van den Heuvel, Brain Center Rudolf Magnus, Department of Psychiatry, University Medical Center Utrecht, Heidelberglaan 100, 3508 GA Utrecht, PO Box 85500, Room: A01.126, The Netherlands; Phone: +31 8875 58244; Fax: +31 8875 55443; Email: m.p.vandenheuvel@umcutrecht.nl 


\section{Abstract}

We introduce comparative connectomics, the quantitative study of cross-species commonalities and variations in brain network topology that aims to discover general principles of network architecture of nervous systems and the identification of speciesspecific features of brain connectivity. Comparing connectomes derived from simple to more advanced species we identify two conserved themes of wiring: The tendency to organize network topology into communities that serve specialized functionality and the general drive to enable high topological integration by means of investment of neural resources in short communication paths, hubs and rich clubs. Within the space of wiring possibilities that conform to these common principles, we argue that differences in connectome organization between closely related species support adaptations in cognition and behavior.

\section{Comparing brains}

Comparative biology and comparative neuroscience generally aim to discover common plans of organization while also accounting for diversity among species. A key objective of comparative studies of brain architecture is to achieve an understanding of the neurobiological basis for the emergence of complex brain structure and function. For example, several classic studies on the cellular composition of the primate cortex have addressed cross-species homologies [1-3] and contemporary comparative analyses have highlighted common cortical phenotypes and important roles of genetic and epigenetic interactions in development for creating cross-species diversity $[4,5]$. Together, these and many other comparative studies have laid the foundations for our understanding of mammalian brain anatomy and function.

One major focus has been on the growing size of brains from smaller to larger animals [6-9] and in particular the significant increase in volume required by the expansion 
of anatomical connections [10-12]. A seminal observation is that the proportion of brain mass spent on cortical white matter follows an allometric scaling relationship between body and brain size across the entire spectrum of simpler to higher-order mammalian species $[13,14]$. In small mammals like the mouse only around $11 \%$ of total brain volume is composed of white matter, in contrast to $27 \%$ in the macaque monkey, $40 \%$ in chimpanzees and $41 \%$ in humans (data taken from [13]). However, despite a larger volume of white matter, maintaining constant connection density among an increasing number of neurons and regions in larger brains will quickly outstrip the volume that can be allocated to longdistance neural wiring [15-17]. Thus the scaling between brain size and white matter volume implies a lower proportion of directly connected neural elements in larger sized brains [15, $17,18]$, making it more and more difficult for neural elements to communicate via direct connections. Maintaining fast and efficient neural communication brings significant benefits to brain function, arguably leading to a fundamental tension or tradeoff [19] between the conservation of neural resources that can be spent on long-distance connectivity and the promotion of efficient communication to support complex neural processing. These apparent opposing or competitive pressures highlight the importance of the topological organization of nervous systems that must provide an arrangement of neural elements and connections to balance the amount of neural resources used for connectivity while at the same time enabling effective information transfer in the service of brain function.

\section{Comparative connectomics}

The examination of brain network topology is a core element of the field of 'connectomics' [20], the emerging science of structural and functional brain networks [21-24]. The increasing availability of connectomes of multiple animal species (Figure 1) opens up a new opportunity for the comparative analysis of network architecture across species. The goal of this review is to introduce "comparative connectomics", defined as the comparison of the 
topological layout of nervous systems across species, with the aim of identifying common principles and variations in network features. Comparative connectomics can provide insight into general principles of neural wiring that apply across species and examine to what extent variations in connectivity between species may form the basis for differences in brain function. As we will discuss, connectomes of different species reconstructed by a broad range of methodologies (see box 1) can be compared by applying a consistent set of network analysis measures and graph analytical tools (see box 2) to enable quantitative comparison of the topological architecture of the nervous systems across and between species. We begin with an overview of currently available systems-level connectome maps, ranging from invertebrates such as Caenorhabditis elegans (a nematode or roundworm) and Drosophila melanogaster (fruit fly), to the macroscale connectome maps of mammalian species such as rodents, old-world monkeys, great apes and humans (Figure 1). Using graph theory as a general framework to quantify topological features of network organization, we first identify common aspects of brain network topology. We then turn to differences in neural wiring and brain network organization among closely related species suggesting that variations in connectome topology subserve species-specific behavioral and cognitive adaptations.

\section{Connectomes from Simpler to More Advanced Animals}

Nearly 30 years ago, serial electron microscope reconstructions of the nervous system of the hermaphrodite nematode Caenorhabditis elegans [25] served as the foundation for the assembly of a near-complete neuronal connectivity network of 890 gap junctions, 6393 chemical synapses and 1410 neuromuscular junctions among 279 neurons of the worm's somatic nervous system [26]. This seminal work inspired later efforts to map the posterior nervous system of the C. elegans adult male [27], as well as the reconstruction of (partial) 
neuronal connectomes of other worm species, including that of the roundworm Pristionchus pacificus [28] and the marine worm Platynereis dumerilii [29].

Early graph theoretical analyses showed the C. elegans nervous system to exhibit a small-world network organization [30] with high clustering (see box 2) and short paths, indicating preferred connectivity into locally clustered circuits, combined with the presence of relatively few long-distance connections that mediate topological short-cuts and facilitate efficient global communication [31]. Further analyses disclosed preferential formation of specific local motifs [26] and a community structure of densely intra-connected and sparsely inter-connected sub-networks or modules [32], an organization that roughly aligns with a classification of neurons into sensory, motor and interneuronal categories [26](Figure 2).

Network analyses of the $C$. elegans connectome have played a prominent role in discussions of wiring minimization models [33, 34], which advocate that the conservation of wiring governs the spatial placement of neuronal elements and accounts for the formation of spatially localized circuits. However, these analyses have also revealed that some neurons and their connections deviate from optimal spatial placement as predicted by a strict minimization of wiring cost $[33,35,36]$, which argues for other, non-geometric biological factors to play a role in the topology of the C. elegans nervous system. Indeed, one of the most prominent features of the adult worm's connectome is the non-uniform distribution of synaptic connectivity (i.e. node degree) across neurons $[26,37]$ with the majority of synaptic connections maintained by a small set of highly connected neurons. These hub neurons maintain dense interconnectivity with each other despite being spatially distributed in both the anterior and posterior extremities of the worm's body. These findings are indicative of a biologically expensive rich club organization of the adult C. elegans connectome in which high-degree elements form a densely centralized core [32]. Interestingly, hub neurons constituting the rich club of the adult C. elegans had previously 
been categorized as command interneurons with known functional importance for information integration [26], coordinated movement and adaptive behaviors of the animal [32]. Furthermore, in C. elegans, these hub neurons have been noted to appear as one of the first elements of the nervous system [38], suggesting a central role of rich club architecture in nervous system development [32, 39].

Other projects aiming at neuron-scale connectivity maps have focused on the reconstruction of the connectome of Drosophila, for example by imaging a sample of approximately 12,000 of the $>100,000$ neurons that make up the adult fly brain [40]. Grouping of individually imaged neurons into over 40 morphologically distinguishable brain areas resulted in a detailed interregional connectome map [40]. Subsequent refinement of this map and detailed graph theoretical analysis showed several highly connected hub regions as well as five network communities (modules) of densely interconnected regions [41]. These five communities largely correspond to known olfactory, visual, mechanosensory/auditory and premotor systems of the fly brain, suggesting that their topological structure forms the anatomical substrate for specialized functional processing (Figure 2). Wiring economy has been suggested to play a major role in local neuron and connectivity placement in the Drosophila brain [42]. However, as in C. elegans, a modular structure minimizing the expense of wiring is supplemented by the presence of a highly connected rich club [41]. Rich club regions are found distributed across all modules of the Drosophila nervous system and their remote geometric placement implies a high cost of wiring of their interconnecting pathways [41](Figure 3). Parallel mapping efforts employing electron microscopy serial sectioning and reconstruction of neuronal circuits in the Drosophila larva have provided detailed wiring diagrams of multisensory circuits that are similar to those of the adult fly brain in demonstrating complex patterns of convergence to enable multimodal information integration [43]. These and other studies establish important 
links between the topology of neuronal circuits and the sensory/behavioral functions of the brain.

Reconstructions of whole-brain connectome maps of more complex species predominantly involve the tracking of macroscale axonal projections between large-scale brain areas. Such reconstructions include mappings of the avian pigeon brain, revealing a modular network architecture and densely connected hub areas [44], as well as detailed mappings of the macroscale systems of several rodent and primate species. Recent comprehensive mapping efforts utilized a large number of injections of anterograde tracers combined with high-throughput serial tomography optical imaging for a detailed reconstruction of the mouse connectome [45]. Although such automated reconstruction procedures will inevitably be prone to some level of measurement error and statistical noise [45], standardized experimental conditions allowed tracking of an unprecedented number $(>15,000)$ of directed projections between over 200 areas of the mouse brain. A parallel effort involved the detailed mapping of over 600 macroscale cortico-cortical pathways combining both anterograde and retrograde tracer experiments [46]. Network analysis of these mouse connectome maps revealed dense local clustering of wiring and modular organization, combined with the existence of topologically short pathways [45, 46]. Furthermore, the strength of anatomical connectivity was noted to approximate a log-normal distribution ranging over 5 orders of magnitude [45]. Projection strengths of anatomical pathways generally were strongest between spatially neighboring regions and decayed monotonically as a function of increasing connection distance. This argues in favor of an important role of geometric factors in shaping the topology of mouse wiring [45]. However, similar to the invertebrate species considered earlier, wiring cost of the mouse connectome is not strictly minimized and its modular organization is complemented by the existence of high-degree rich club hubs mediating biologically expensive (long distance) connections between modules [47, 48] (Figure 3). Generative modeling approaches proved successful in 
reproducing the modular aspects of connectome topology of the mouse brain simply by minimizing wiring cost, but these models failed to account for the emergence of longdistance connections until the penalty imposed on spatial distance was adaptively relaxed for higher degree hubs [47].

A different approach aiming to build a rat cortical connectome involved collating data across $>16,000$ literature reports of tract tracing experiments. Collectively, these data captured 1,923 cortical association macro-connections spanning 73 gray-matter areas of the rat cerebral cortex, together with an ordinal assessment of connection strength [49]. Graph theoretical examination revealed short communication paths, high clustering, connected communities largely coinciding with functionally specialized systems [49], and a central rich club $[49,50]$ comprising a set of highly connected, spatially distributed and multimodal cortical areas [49] (Figure 3).

Consensus matrices obtained by collating data across large numbers of tracing experiments have also been built for the cat [51], ferret [52] and macaque brains [53]. One of the earliest mappings of a mammalian cerebral system involved the compilation of the macroscale cat connectome, describing 65 cortical areas and $>1000$ cortico-cortical pathways [51]. Network analysis of the cat connectome showed short length paths and strong clustering of anatomical wiring within functionally specialized motor, visual, auditory and fronto-limbic subsystems [54]. Follow-up analysis identified different types of hub areas in the cat cortex [55], and defined a central, reciprocally interconnected core module $[56,57]$ corresponding to a rich club of cortical hubs [58].

The systematic analysis of anatomical connectivity patterns in the macaque brain was propelled forward by the early connectomics pioneer Rolf Kötter (1961-2010) who founded the open-access Collation of Connectivity Data for the Macaque (CoCoMac) database, which aggregated data from hundreds of macaque tract-tracing experiments [53, 59]. Graph theoretical analyses of macaque connectome maps revealed high clustering, a 
hierarchical ordering of wiring from primary to multimodal areas [60], short path lengths, pronounced modular organization [61-63], as well as the presence of spatially distributed but centrally connected cortical hub areas $[55,62]$ (Figure 3 ). As already noted by early computational analyses of macaque interareal connectivity [64], inverse correlations between projection density and geometric length suggest an important role of spatial or geometric factors in shaping the topology of macaque interareal projections [65]. However, modeling analyses of macaque connectivity have also pointed out an important role for topological factors in shaping macaque connectivity, e.g. the drive to enable efficient communication pathways by means of the formation of high-cost long-distance projections [35] and the high investment of connectivity around hubs and a rich club core $[55,66,67]$. These network attributes are difficult to account for by purely geometric factors.

The development of diffusion-weighted imaging combined with tractography has allowed for the assembly of the macroscale human connectome [68-72]. Despite the caveats and limitations regarding the interpretation of the diffusion MRI signal [73-75] and its use in connectome reconstruction (see for discussion [68, 76-78]), diffusion weighted imaging is currently one of the most widely used methods for the assessment of anatomical connectivity in the human brain. Several large-scale efforts, including the Human Connectome Project [79], are delivering unprecedented amounts of high-quality human connectome data, and advances in ex vivo imaging techniques might provide even more detailed maps of post mortem animal and human wiring in the future (see Box 1). Paralleling key features of connectome topology already reviewed for other animals, network analysis of the human connectome has shown an organization of densely connected communities that form the anatomical wiring skeleton of known functional domains [8085](Figure 2). This modular structure is complemented by anatomically long-distance projections that support topologically short-distance global communication paths $[68,69]$, 
combined with densely connected and topologically central communication hubs $[68,71$, 86-88] that form a central rich club in the human brain [39, 89-92] (Figure 3).

Having surveyed our current knowledge of connectome topology across a range of species, we are now in a position to examine the potential principles that may drive common themes in wiring patterns, as well the important roles of cross-species variations in differentiating behavioral and cognitive adaptations.

\section{Common Principles of Connectome Wiring}

We first discuss which general principles may underlie common themes of connectome organization observed across species. Across the range of species studied so far, we observe a strong tendency at both micro and macro scales of network organization for neurons (micro) or brain regions (macro) to connect to their spatial and topological neighbors, favoring the formation of spatially co-localized, topologically clustered cliques or communities [80] (Figure 2). This community architecture largely coincides with the formation of physiologically specialized functional domains in nervous systems, consistent with the idea that anatomical wiring plays an important role in the functional differentiation of cortical areas [93-95]. Cross-species comparison shows a high level of consistency of functional domains across species, with human, macaque and rodents showing several homologous primary and higher-order associative limbic and cognitive networks [96-99]. The conservation of wiring has long been proposed as a fundamental rule governing the local layout of circuits $[34,47,64,65,100-102]$. Geometric constraints promote the emergence of functionally specialized network communities in nervous systems. Across species, it has been hypothesized that the growth of brain volume across species favors the existence of local modules $[103,104]$ while penalizing the formation of long-range connectivity as such connections become more and more expensive in terms of neural 
resources. Support for such notions come from studies examining the volume of white matter connectivity tracts across a wide range of primate species, revealing long-distance (and thus costly) connectivity in the corpus callosum to be lower in larger primate brains, while intra-hemispheric white matter volume is enlarged [105]. A more local organization of cortical wiring in larger brains may promote the emergence of specialized brain functionality in larger animals [103, 104, 106-108]. For example, a stronger modularity structure of connectome wiring may have played a role in the formation of more spatially localized cortical fields for visual, sensory and motor information processing in larger mammalian brains [109], as well as increased brain lateralization, preferred hand-use, and the development of specialized traits like language processing in humans [110-112].

A second general theme of connectome topology includes the drive of nervous systems to invest resources in network attributes that bring topological integration (Figure 3). Across the range of examined species, all nervous systems show topologically short and efficient paths, regardless of increasing brain network size. Although modeling studies have generally succeeded in accounting for parsimonious local wiring and cost-controlled community structure, they have difficulty explaining the formation of long-distance pathways (essential for efficient communication paths) unless constraints on wiring cost are relaxed [47, 101, 102, 113]. Across species, the consistent presence of long-distance connections that violate strict minimization of wiring suggests a general drive of neural systems to invest neural resources in network attributes that maintain short communication relays.

The strong drive to invest costly resources in network attributes that bring topological integration may become even more apparent from the consistent allocation of neural assets to the formation of a densely connected core or rich club [32, 87, 114]. This general tendency to centralize connectivity appears to be ubiquitous: it is observed across spatial scales (e.g. from patterns of information flow in microcircuits [115-117] to whole- 
brain systems), as well as across small and large nervous systems of vastly different species (see also Box 3).

Communication in nervous systems goes beyond the simple relaying of messages along shortest paths and involves continual processing of information at each neural step. The centralization of neural connectivity into a connective core may thus bring important benefits with regard to information integration $[32,87,118,119]$, potentially facilitating higher-order brain functionality that thrives on integrated information, such as coordinated movement in the worm [32] and/or executive functioning in humans [120]. Due to their central embedding in network topology, hubs and their connections can attract and disseminate a large proportion of all neural communication [89, 119], forming an anatomical substrate for the exchange of information between otherwise segregated domains. This has led to the theoretical idea that hubs and rich clubs provide an anatomical infrastructure [87] for the formation of a "global workspace" [121-123] or "connective core" [124], a functional construct in which information from different parts of the system is integrated. These ideas are supported by observations demonstrating that hub regions in mammalian species tend to overlap with multimodal association cortex $[125,126]$, display an intricate neuronal architecture with heavily branched pyramidal neurons [127-131], pose high energy demands $[19,90,132]$, and form convergence zones between different functional networks [133-135].

The common theme across nervous systems to invest neural resources in network attributes that bring topological integration argues for a more diverse set of principles than strict conservation of neural resources to shape the connectivity layout of nervous systems $[136,137]$. Rather, the drive to invest neural resources in dense connectivity around an integrative core appears to favor the emergence of connectome attributes that are advantageous for enabling short pathways and transmodal communication. Offsetting their greater biological cost in terms of neural and metabolic resources, these topological features 
may bring strong potential benefits for integrative neural processing and cognitive brain function and behavior.

\section{Variations Support Behavioral and Cognitive Adaptations}

Although we argue for common cross-species themes in connectome organization, a proposed tradeoff between pressures to conserve wiring cost and pressures to maximize topological integration $[19,138]$ cannot, by itself, account for the abundant diversity in brain connectivity and nervous system organization across species. Multiple factors, including variations in environmental factors, genes and genetic regulatory networks operating during development combine to generate a diverse set of brain networks even across closely related species. A natural next question, then, is how differences in connectome wiring between species relate to species-specific differences in behavior and information processing. This question is most productively explored by comparing connectomes of relatively closely related species that share a basic anatomical plan and an overlapping behavioral repertoire.

One example is offered by comparative studies of the nervous systems of roundworms. Comparison between reconstructions of microscale neural networks of the pharyngeal systems of C. elegans and P. pacificus showed significant differences in neural connectivity that could be linked to their distinct feeding behaviors [28]. P. pacificus, in contrast to the bacterial feeder C. elegans, is a predator with a distinct anatomy of its mouth areas. Side-by-side comparison of the layout of synaptic connectivity of the pharyngeal systems of the two species revealed significant differences [28] (Figure 4), with P. pacificus showing increased connectivity around neural elements controlling tooth-like denticles. Comparative network centrality analysis further showed that presumptive communication paths and information flow in P. pacificus are much more tuned towards the control of pharyngeal motor neurons. Thus, differences in connectome topology appear to constitute at 
least part of the biological basis for the substantial divergence in feeding behavior between the two worm species [28]. In addition, a second comparative example in the roundworm (not across species but gender) is provided by dimorphic differences in neural wiring of the reproductive system of $C$. elegans. Male worms include 383 neurons (in comparison to 302 of hermaphrodites) with dimorphic changes in neurons and connectivity mostly focused around the formation of circuits related to mating and reproduction [25, 27]. Male worms, but not hermaphrodites, are capable of sexual adaptive learning that facilitates effective mate finding and, interestingly, a recent study revealed a key role of specific neurons and their wiring in the emergence of this behavior [139]. Detailed reconstruction of synaptic connectivity revealed two newly discovered neurons to be incorporated in existing circuits during late development, with the newly added connectivity and accompanying change in network structure allowing the male worm to add sex-specific learning plasticity to its functional repertoire [139].

Comparative connectivity analyses between primate species have similarly argued for a relationship between subtle differences in connectome wiring and divergent behavior [140]. For example, bonobos and chimpanzees share a recent common ancestor but they show quite distinct social interactive behavior, with bonobos showing less aggression and higher social tolerance as compared to chimpanzees (see [140, 141] for a discussion). Comparative analysis of MRI-derived reconstructions of brain connectivity between the two species shows anatomical connectivity between the amygdala and anterior cingulate regions, a network of subcortical and cortical areas involved in the modulation of social and emotional behavior [142, 143], to be stronger in bonobo apes than in chimpanzees [140]. Furthermore, comparative MRI studies between macaques, chimpanzees and humans [110], have linked elaboration of anatomical connectivity of the arcuate fasciculus connecting temporal and frontal cortical areas to the development of language and speech processing in humans (Figure 4) [110, 144]. 
In addition to evidence for behaviorally-related connectivity differences in specific circuits, comparative connectome examinations between primates and humans have further offered support for a role of variations in system level connectivity patterns in the evolutionary emergence of specific advanced brain functions. Although studies have reported a general homology of connectome organization across macaque, chimpanzee and human, there is evidence for significant species-specific differences in the wiring of parietal and medial prefrontal regions $[67,145]$. Comparative connectome analyses suggest that areas of the frontal cortex in humans exhibit a less central position as compared to homologous areas in macaques and chimpanzees [67, 104]. In addition, functional connectivity hubs in the human brain have been argued to exhibit a more spatially distributed organization compared to non-human primates [146], differences that may contribute to the increasing functional specialization of frontal cortical areas [104]. Potentially more, and more centrally connected hubs in other parts of the cortex may confer higher robustness and bring greater support for the functional involvement of other areas in global processing [146]. These thoughts are supported by recent comparisons between chimpanzees and humans, which revealed strong expansion of the precuneus in humans [147]. Indeed, the precuneus is one of the most central and most connected hub areas of the human brain $[68,114]$. These ideas parallel observations that variations in connectome organization and hub wiring relate to individual variation in cognitive processing in humans $[120,148-152]$.

\section{Concluding remarks and future directions}

The central idea of this review is that nervous systems of different species exhibit both common themes and important variations in connectome organization. We argue that connectome architecture may follow general principles of wiring, shaped by forces that minimize the expenditure of biological resources and forces that favor functionally 
important topological attributes that benefit efficient communication and global integration. A competition and trade-off between these opposing forces can account for the ubiquity of connectomes that are generally parsimoniously wired while allowing for features that can promote topological integration. We further argue that, within the envelope of wiring solutions that conform to these general principles, subtle variations in connectome organization support species-specific adaptations in behavior and cognitive functioning.

The ideas put forth in this review, in particular our thoughts on adaptive changes of network attributes to form biological underpinnings of variations in brain function, of course need thorough empirical testing. Comparative studies that employ uniform acquisition methodology to map (see box 1) and study (see box 2) connectomes across species are needed to rigorously map commonalities and differences of connectivity patterns. Furthermore, investments in studies that explicitly address the diversity of brain architectures are needed to draw more secure inferences about the putatively universal principles shaping connectome evolution. This would require examinations across a much wider range of species than currently available. For example, besides the work of reconstructing connectomes of nematode, insect, avian and mammalian species, as summarized in this review, comparative connectomics could be extended further to encompass the nervous systems of animals with a completely different body plan but who may nevertheless share universal attributes of connectome organization (see box 3). In addition to the collection of more comprehensive and consistent empirical data, this burgeoning field would benefit from computational modeling studies that simulate generative mechanisms behind cross-species network evolution and test the roles of network attributes with respect to functional diversity [118], dynamics [83, 153], resilience and integration $[154,155]$.

In focusing on patterns of brain connectivity, comparative connectomics is subject to several important limitations. First, today's comparative connectomics is constrained by the 
divergent methodologies used to assess connectivity at different scales and in different species (e.g. electron microscopy, tract-tracing, MRI, see box 1). This limitation underscores the importance of finding ways to reconstruct connectomes more uniformly across different nervous systems. Second, it is important to note that the architecture of a nervous system must be considered in the context of the overall structural anatomy, physiology and ecological embedding of the organism [156]. Among many other factors, an organism's body plan, the arrangement and physiology of its sensory apparatus, and the geometry of its musculoskeletal system, are obviously important factors that shape, constrain and enable behavioral (and cognitive) function, as well as the layout of the nervous system. The common themes of connectome organization highlighted in this review cut across vast differences in body plan and "evolutionary complexity". This suggests that the observed principles of wiring reflect universal features of efficient and effective network organization that cause common connectome patterns to be found across a wide range of species. Viewed from another perspective, they may define hard limitations to connectome formation that cannot easily be contravened by natural selection. The competitive pressures of parsimonious wiring and topological integration impose general trajectories and boundaries that define the space of network topologies that are geometrically and functionally possible. This space is embedded within a "theoretical morphospace" of biological forms [157] and defines the realm within which connectome topologies can vary, at least in principle, and contribute to adaptations that support different behavioral and cognitive specialization. The size and shape of this common morphospace, how it constrains the possible behavioral and cognitive repertoire [158-160], and how it compares to the total morphospace of natural and human-engineered networks (see box 4) all remain important open questions. 
We hope that, with growing access to connectome data from a range of species, comparative connectomics may become a useful addition to the spectrum of approaches aiming to account for cross-species commonalities and differences in brain structure and function. 


\section{Box 1. Connectome reconstruction methods}

Different methodologies can be applied for the reconstruction of connectome maps. Electron microscopy techniques have enabled reconstruction of the $C$. elegans nervous system at the synaptic level [25]. Tracer injection techniques combined with high-throughput serial tomography optical imaging have enabled a high-resolution reconstruction of the mouse connectome [45] and systematic collations of data across high numbers of tracing experiments have resulted in grouped consensus matrices of the cat, macaque and rat brains. Advances in in vivo diffusion MRI techniques have made it increasingly feasible to reconstruct macroscale connectomes of individual brains of great apes [145] and humans. The development of techniques like CLARITY [161] and 3D Polarized Light Imaging (3D-PLI) may bring unprecedented high resolution reconstructions of animal and human connectome wiring in the near future.

Today, the field of comparative connectomics is limited by the fact that connectome maps of different species are being acquired with different types of methodology, restricting direct comparison of connectomes across a wide range of species. For example, the C. elegans connectome describes neuron-to-neuron interactions at the microscale, while the macaque and cat connectome describe region-to-region connections at the macroscale level of nervous system organization. In addition, while electron microscopy, tract-tracing and MRI-derived connectome reconstructions can provide information on the strength of connections (in graph theoretical analysis often referred to as 'weighted networks'), connectome maps derived from cumulative collation of data across published literature are often limited in only providing information on the presence or absence of connections (referred to in connectome literature as 'binary networks') or coarse categorization of the magnitude of pathways.

We thus argue that there are two aspects that are critical for enabling comparative studies in the future. First, it is crucial to establish ways to interpolate across different methodologies, ideally by directly comparing brain connectivity patterns derived by multiple techniques in the same organism, for example tract tracing and noninvasive neuroimaging [76, 162, 163]. Second, the 
application of the same technique to multiple species (e.g., $[28,145])$ facilitates cross-species comparisons.

Box 2. Graph theory. The connectomes of different species can be compared by applying a consistent set of network analysis methods. These methods are principally drawn from the mathematical field of graph theory, enabling quantitative comparison between species using the same mathematical language. Within this approach a nervous system is described as a graph, consisting of a collection of nodes (e.g. neurons, brain areas) and a collection of edges describing the pairwise relationships between nodes (e.g. synaptic connections, macroscopic axonal projections)(Figure I). Once such a mathematical description of a network is made, graph theoretical metrics can be used to describe -and, across species, compare-topological properties of the network. The metric of degree represents the number of connections attached to a node of the network. Clustering describes the tendency of nodes to form closed triangles, such that the nearest neighbors of a node are also directly connected to each other. The metric of clustering reflects the tendency of a network to form topologically local circuits, and is often interpreted as a metric of information segregation in networks. A path describes a route of information transfer between two nodes in a network, with the metric of path length describing the number of steps (comprising unique edges) crossed when traveling from one node to another node in a network. The shortest path length expresses the minimal number of steps needed to travel between nodes, and is often interpret as a metric of the efficiency of information transfer between nodes of a network. Hubs are nodes with a high degree and a topologically central position in the overall network, with the core of a network describing a set of highly connected nodes that are mutually densely connected. The related concept of rich club organization describes the propensity of highly connected nodes to be more densely connected to each other than expected based on the individual degree of the nodes. A network's community structure refers to the tendency of a network to form densely connected subgraphs or modules, corresponding to a subset of nodes that are densely connected to each other 
and sparsely connected to nodes in other modules. In nervous systems, anatomical modules often overlap with known functional systems.

\section{Box 3. Nerve nets}

The nematode, insect, avian and mammalian species discussed in this review all describe species that display a central brain system. Others, like jellyfish, hydra and starfish do not have a centralized brain. Instead, their nervous system comprises as a 'nerve net' (Figure IIA) with neurons distributed across most parts of the animal, a type of system organization reflecting their distinct body plan $[164,165]$. The nervous systems of jellyfish and hydra are often described as a simple network in which neurons are only connected to their spatial neighbors (in network terms this would make a simple 'regular graph' or spatial 'grid' with no modules, short paths or hubs).

Recent studies have however suggested that the nervous systems of adult jellyfish and hydra may exhibit a much more diverse architecture, including potential concentration of neural elements into complex structures facilitating information integrative processes [165-167]. The nervous systems of adult jellyfish consist of multiple components (see [164] for an introduction on jellyfish nervous systems), including sensory structures (rhopalia, Figure IIB) that are connected via an interconnecting nerve ring(s) system running around the bell (Figure IIB). This nerve ring is involved in integrating the swimming, visual and tentacle system [165] and argued to represent a rudimentary central nervous system [168, 169].

Thus although the basic plan of the nerve nets of species like jellyfish and hydra may be laid out quite differently from the nervous systems of the species discussed in this review, we argue that there may be common topological attributes, for example a centralized system of interconnected neuronal structures. This suggestion calls for further expansion of the currently available set of connectomes beyond those discussed in this review, and emphasizes the need for comparative connectomics to examine commonalities and differences in nervous system architecture across a (much) wider range of species. 


\section{Box 4. Comparative analysis of different classes of networks}

Going beyond the examination of shared topological features across nervous systems, the generalized mathematical language of graph theory also offers tools for the comparison of the organization of brain networks to other classes of networks studied by very different scientific disciplines. Many real-world systems operate as some sort of interaction or communication network, including for example social networks, gene regulatory networks, computer networks and transportation networks. Similar to brain networks, many of these real-world networks display an efficient small-world organization, a pronounced community structure with densely connected modules, as well as the formation of hubs and rich clubs [30, 37, 170]. Going beyond the comparison of networks within the class of nervous systems, the field of 'comparative network analysis' examines commonalities and differences across a broad range of network classes.

A core concept in this context is that of network morphospace [157], which examines underlying morphological characteristics of networks by describing common and differentiating aspects across networks compared to the total space of possible networks. Applications of morphospace analysis include examinations of the capacity of brain networks to diffuse and route signals in comparison to social, gene regulatory and email networks [171]. A complementary approach is based on applications of spectral graph theory [172], with the spectrum describing the multiset of eigenvalues of a network's adjacency matrix. Networks showing overlapping spectra display common organizational and functional features. For example, spectral examinations have shown similar features between nervous systems and the organization of ecological networks, an observation that may provide clues to selection pressures playing a role in the evolution of both systems [173].

Comparative network analysis allows for the examination of commonalities and differences between classes of networks. In particular, it provides a powerful approach to answer the question of which topological network attributes are specific to nervous systems and which represent more 
universal properties of network organization [174]. As such, it provides a unique tool to examine which network properties are unique to brain networks, and which properties are shared with other types of communication and interaction networks.

\section{Glossary}

Allometric scaling: relationship between body size and shape, morphometry and function of brain parts across species where one or more of these measures change exponentially or nonlinearly

Adjacency matrix: A systematic description of the absence or presence of a connection or edge between all pairs of nodes of a network, represented in the form of a square matrix. Association matrix: A summary of the absence or presence (potentially including information on the strength of an association) of all pairwise associations of network nodes, represented in the form of a square matrix.

Brain network: Any set of structural or functional relations among brain elements.

Connectivity: Description of the anatomical projections (e.g. synaptic connections, axonal tracts) between brain network nodes (e.g. neurons, cortical areas).

Connectome: Comprehensive network map of the neural connections of a nervous system. Connectomics: A subfield of neuroscience that studies the reconstruction and analysis of connectomes

Comparative connectomics: the quantitative study of cross-species commonalities and variations in brain network topology

Functional connectivity: statistical relationship between time-series of physiological activity (e.g. fMRI, spike trains) of neural elements (e.g. neurons, brain regions).

Graph: A mathematical description of a network, consisting of a collection of nodes (e.g. neurons, brain areas) and a collection of edges describing the pairwise relationships between nodes (e.g. synaptic connections, macroscopic axonal projections) (see Box 2). 
Homology: Properties of nervous system organization (e.g. cellular architecture, wiring organization) that are shared between species

Morphospace: Originally defined in evolutionary theory as the space of all possible body shapes or morphologies for a given group of organisms

Network: In nervous systems, a network describes the set of neural elements (e.g. neurons, brain regions) and their relationships (e.g. synaptic connections, macroscale pathways, functional interactions).

Rich club organization: the property of a network to display an increasing level of connectivity between subsets of highly connected nodes, a level of connectivity higher than would be expected on the basis of the individual degree of the subset of nodes alone.

Scale-free organization: A class of networks with a degree distribution that follows a power law, or, more generally, a type of broad-scale or fat-tailed degree distribution.

Small-world organization: A class of networks that shows both a high level of clustering (like a regular lattice) and short characteristic paths (like a random graph).

Spectral graph theory: A branch of graph theory that studies networks by examining the spectrum of eigenvalues and eigenvectors of the network's adjacency matrix

Topology: The topological structure of a network describes the arrangement of connections in a network and is invariant to any continuous spatial deformation of the system. 


\section{Figures}

Figure 1. Connectomes across species. The Figure displays reconstructed connectomes of eight different species, including (upper row from left to right, and lower row from left to right) Caenorhabditis elegans (roundworm) [26], Drosophila (fruit fly) [40], pigeon [44], mouse [45], rat [49], cat [51], rhesus monkey (macaque, FE91 atlas) [53, 175] and human (Human Connectome Project data, 220 cortical regions, [129]). Connectomes are represented as connectivity matrices with rows and columns depicting source and target regions (grouping regions participating in the same community together) and with the elements of the connectivity matrices showing the reconstructed projections. Pathways are grouped into weak (blue), medium (yellow) and strong (orange).

Figure 2. Community structure. Studies have shown consistent community organization of nervous systems across species, including (A) the C. elegans neuronal network, and (B) Drosophila, (C) macaque and (D) human connectomes. Anatomical communities obtained by graph theoretical analysis often with known functional domains, as for example the olfactory (yellow), visual (purple and orange, left and right), auditory/mechanosensory (magenta) and pre-motor (red) functional systems of the fly brain (panel B) [41]. Modular decomposition of the human connectome as derived from diffusion weighted imaging revealed the formation of at least six anatomical communities overlapping spatial domains of the human brain. A, C and D reprinted under the creative Commons Attribution License from [26], [62] and [68], B adapted and reproduced with permission from [41].

Figure 3. Hubs and rich club organization across species. Connectome studies have shown hub and rich club organization for (A) the microscale C. elegans nervous system, as well as for the macroscale brain networks of (B) mouse, (C) rat, (D) macaque and (E) human. Analysis of the C. elegans connectome has revealed a small set of highly connected 
rich club hub neurons distributed across anterior (red nodes) and posterior (yellow nodes) parts of the animal. (B) Network analysis of the mouse connectome has shown the existence of highly connected and highly central connector hubs (red nodes), which mediated most of the inter-modular connections between functionally specialized modules of the community structure (nodes are color-coded by modular affiliation). (C) Network analysis of the rat connectome has shown the rich club (red line) to participate across multiple functional domains (depicted as colored blocks). (D) Connectome analysis of the macaque brain has revealed rich club members (red nodes) to be spatially distributed across the cortex. (E) Network studies of the human connectome have similarly shown the existence of highly connected hubs in the human brain (depicted as red, yellow, blue and green regions). These hub areas are distributed across multiple functional domains and show strong rich club organization. A and E adapted and reproduced from [32] and [72] under the Creative Commons Attribution-Noncommercial-Share License, B adapted and reproduced with permission from [47], C adapted and reproduced with permission from [49], D reprinted under the Creative Commons License from [66].

Figure 4. Connectivity comparison across species. (A) A side-by-side comparison of the neuronal organization (upper panel) and wiring (graphs in the lower panel) of the pharyngeal system across two worm species, P. pacificus (right) and C. elegans (left). A comparison between the two systems (consisting of the same number and same types of neurons) reveals wiring differences: the predator P. pacificus shows higher complexity of connectivity around neural elements controlling the toothlike denticles as compared to C. elegans [28]. (B) Comparison of macroscale connectivity between macaque (top), chimpanzees (middle) and humans (bottom) revealed more elaborate connectivity of the arcuate fasciculus in humans, a tract important for complex language processing [110]. (C) Comparison of whole-brain functional connectivity patterns between macaques and humans revealed areas of both weaker (blue areas) and stronger functional connectivity across the cortex 
(red areas) in humans as compared to macaques, as well as overall more spatially diffuse hub structure in humans [146]. A adapted and reproduced with permission from [28], B adapted and reproduced with permission from [110], C adapted and reproduced under the Creative Commons Attribution-Noncommercial-Share License from [146].

Figure Box 2. Graph metrics. Figure illustrates (from left to right) the mathematical description of a network as a graph consisting of nodes and edges, the metric of degree, a communication path, the metric of clustering, the formation of a densely connected rich club or core and the community structure of a network, reflecting the formation of densely connected subgraphs or modules within the network.

Figure Box 3. Jellyfish nerve net. (A) The nervous system of a jellyfish involves a nerve net. (B) Sensory structures (rhopolia) in several jellyfish species include concentrated neural structures that are interconnected by an organized nerve ring ( $\mathrm{RN}$ in $\mathbf{B}$ and also shown in panel $\mathbf{C})$, potentially supporting multiple communication pathways. A adapted and reproduced with permission from [165], B adapted and reproduced with permission from B [164] (RN depicts nerve ring).

\section{Acknowledgements}

We thank Lianne Scholtens, Jim Rilling, Tom Schoenemann for discussions and comments. MPvdH was supported by a VENI (\# 451-12-001) grant from the Netherlands Organization for Scientific Research (NWO) and a Fellowship of MQ.

\section{References}

1 Campbell, A.W. (1905) Cytoarchitectonic maps of the human brain. Histological studies on the localisation of cerebral function. Cambridge: University Press

2 Brodmann, K. (1909) Vergleichende Lokalisationslehre der Grosshirnrinde. Johann Ambrosius Barth

3 von Bonin, G. and Bailey, P. (1947) The neocortex of Macaca mulatta Univ. of Illinois Press 
4 Northcutt, R.G. and Kaas, J.H. (1995) The emergence and evolution of mammalian neocortex. Trends in neurosciences 18, 373-379

5 Krubitzer, L. (2007) The magnificent compromise: Cortical field evolution in mammals. Neuron 56, 201-208

6 Jerison, H.J. (1973) Evolution of the Brain and Intelligence. Academic Press

7 Herculano-Houzel, S., et al. (2007) Cellular scaling rules for primate brains. Proceedings of the National Academy of Sciences of the United States of America 104, 3562-3567

8 Deaner, R.O., et al. (2007) Overall brain size, and not encephalization quotient, best predicts cognitive ability across non-human primates. Brain Behav Evolut 70, 115-124

9 Schoenemann, P.T. (2013) Hominid Brain Evolution. In A Companion to Paleoanthropology

(Begun, D.R., ed), Blackwell Publishing

10 Schoenemann, P.T. (2006) Evolution of the size and functional areas of the human brain. Annu

Rev Anthropol 35, 379-406

11 Rilling, J.K. and Seligman, R.A. (2002) A quantitative morphometric comparative analysis of the primate temporal lobe. J Hum Evol 42, 505-533

12 Schoenemann, P.T., et al. (2005) Prefrontal white matter volume is disproportionately larger in humans than in other primates. Nature neuroscience 8, 242-252

13 Zhang, K. and Sejnowski, T.J. (2000) A universal scaling law between gray matter and white matter of cerebral cortex. Proceedings of the National Academy of Sciences of the United States of America 97, 5621-5626

14 Hofman, M.A. (1988) Size and shape of the cerebral cortex in mammals. II. The cortical volume. Brain, behavior and evolution 32, 17-26

15 Hoffman, M.A. (2014) Evolution of the human brain: when bigger is better. Frontiers of Neuroanatomy 8

16 Wang, S.S., et al. (2008) Functional trade-offs in white matter axonal scaling. J Neurosci 28, 4047-4056

17 Ringo, J.L. (1991) Neuronal interconnection as a function of brain size. Brain, behavior and evolution $38,1-6$

18 Herculano-Houzel, S., et al. (2010) Connectivity-driven white matter scaling and folding in primate cerebral cortex. Proceedings of the National Academy of Sciences of the United States of America 107, 19008-19013

19 Bullmore, E. and Sporns, O. (2012) The economy of brain network organization. Nature reviews $13,336-349$

20 Sporns, O., et al. (2005) The human connectome: A structural description of the human brain.

PLoS computational biology 1, e42

21 Sporns, O. (2011) Networks of the Brain MIT Press, Cambridge

22 Bullmore, E. and Sporns, O. (2009) Complex brain networks: graph theoretical analysis of structural and functional systems. Nature reviews 10, 186-198

23 van den Heuvel, M.P. and Hulshoff Pol, H.E. (2010) Exploring the brain network: a review on resting-state fMRI functional connectivity. European neuropsychopharmacology : the journal of the European College of Neuropsychopharmacology 20, 519-534

24 Fornito A, Z.A., Bullmore ET (2016) Fundamentals of Brain Network Analysis. Academic Press, San Diego

25 White, J.G., et al. (1986) The structure of the nervous system of the nematode Caenorhabditis elegans. Philosophical transactions of the Royal Society of London. Series B, Biological sciences $314,1-340$

26 Varshney, L.R., et al. (2010) Structural properties of the Caenorhabditis elegans neuronal network. PLoS computational biology 7, e1001066

27 Jarrell, T.A., et al. (2012) The connectome of a decision-making neural network. Science 337, 437-444

28 Bumbarger, D.J., et al. (2013) System-wide rewiring underlies behavioral differences in predatory and bacterial-feeding nematodes. Cell 152, 109-119 
29 Randel, N., et al. (2014) Neuronal connectome of a sensory-motor circuit for visual navigation. Elife 3

30 Watts, D.J. and Strogatz, S.H. (1998) Collective dynamics of 'small-world' networks. Nature $393,440-442$

31 Latora, V. and Marchiori, M. (2001) Efficient behavior of small-world networks. Phys Rev Lett 87

32 Towlson, E.K., et al. (2013) The rich club of the C. elegans neuronal connectome. J Neurosci 33, 6380-6387

33 Chen, B.L., et al. (2006) Wiring optimization can relate neuronal structure and function.

Proceedings of the National Academy of Sciences of the United States of America 103, 4723-4728

34 Cherniak, C. (1994) Component Placement Optimization in the Brain. Journal of Neuroscience $14,2418-2427$

35 Kaiser, M. and Hilgetag, C.C. (2006) Nonoptimal component placement, but short processing paths, due to long-distance projections in neural systems. PLoS computational biology 2, e95

36 Ahn, Y.Y., et al. (2006) Wiring cost in the organization of a biological neuronal network.

Physica A 367, 531-537

37 Barabasi, A.L. and Albert, R. (1999) Emergence of scaling in random networks. Science 286, 509-512

38 Varier, S. and Kaiser, M. (2011) Neural development features: spatio-temporal development of the Caenorhabditis elegans neuronal network. PLoS computational biology 7, e1001044

39 van den Heuvel, M.P., et al. (2015) The Neonatal Connectome During Preterm Brain Development. Cerebral Cortex 25, 3000-3013

40 Chiang, A.S., et al. (2011) Three-dimensional reconstruction of brain-wide wiring networks in Drosophila at single-cell resolution. Current biology : $C B$ 21, 1-11

41 Shih, C.T., et al. (2015) Connectomics-based analysis of information flow in the Drosophila brain. Current biology : $C B$ 25, 1249-1258

42 Rivera-Alba, M., et al. (2011) Wiring Economy and Volume Exclusion Determine Neuronal Placement in the Drosophila Brain. Current Biology 21, 2000-2005

43 Ohyama, T., et al. (2015) A multilevel multimodal circuit enhances action selection in Drosophila. Nature 520, 633-U107

44 Shanahan, M., et al. (2013) Large-scale network organization in the avian forebrain: a connectivity matrix and theoretical analysis. Frontiers in computational neuroscience 7

45 Oh, S.W., et al. (2014) A mesoscale connectome of the mouse brain. Nature 508, 207-214

46 Zingg, B., et al. (2014) Neural networks of the mouse neocortex. Cell 156, 1096-1111

47 Rubinov, M., et al. (2015) Wiring cost and topological participation of the mouse brain connectome. Proceedings of the National Academy of Sciences of the United States of America 112, 10032-10037

48 van den Heuvel, M.P. and de Reus, M.A. (2014) Chasing the dreams of early connectionists.

ACS chemical neuroscience 5, 491-493

49 Bota, M., et al. (2015) Architecture of the cerebral cortical association connectome underlying cognition. Proceedings of the National Academy of Sciences of the United States of America 112, E2093-2101

50 van den Heuvel, M.P., et al. (2015) Topological organization of connectivity strength in the rat connectome. Brain structure \& function Epub ahead of print

51 Scannell, J.W., et al. (1995) Analysis of connectivity in the cat cerebral cortex. J Neurosci 15, 1463-1483

52 Sukhinin, D.I., et al. (2015) Building the Ferretome. bioxiv http://dx.doi.org/10.1101/014134 53 Stephan, K.E., et al. (2001) Advanced database methodology for the Collation of Connectivity data on the Macaque brain (CoCoMac). Philosophical transactions of the Royal Society of London. Series B, Biological sciences 356, 1159-1186 
54 Hilgetag, C.C., et al. (2000) Hierarchical organization of macaque and cat cortical sensory systems explored with a novel network processor. Philosophical transactions of the Royal Society of London. Series B, Biological sciences 355, 71-89

55 Sporns, O., et al. (2007) Identification and classification of hubs in brain networks. PloS one 2, e1049

56 Zamora-Lopez, G., et al. (2009) Graph analysis of cortical networks reveals complex anatomical communication substrate. Chaos (Woodbury, N.Y 19, 015117

57 Zamora-Lopez, G., et al. (2011) Exploring brain function from anatomical connectivity.

Frontiers in neuroscience 5, 83

58 de Reus, M.A. and van den Heuvel, M.P. (2013) Rich Club Organization and Intermodule Communication in the Cat Connectome. Journal of Neuroscience 33, 12929-12939

59 Bakker, R., et al. (2012) CoCoMac 2.0 and the future of tract-tracing databases. Frontiers in neuroinformatics 6, 30

60 Felleman, D.J. and Van Essen, D.C. (1991) Distributed hierarchical processing in the primate cerebral cortex. Cereb Cortex 1, 1-47

61 Sporns, O., et al. (2000) Theoretical neuroanatomy: relating anatomical and functional connectivity in graphs and cortical connection matrices. Cereb Cortex 10, 127-141

62 Modha, D.S. and Singh, R. (2010) Network architecture of the long-distance pathways in the macaque brain. Proceedings of the National Academy of Sciences of the United States of America $107,13485-13490$

63 Shen, K., et al. (2012) Information processing architecture of functionally defined clusters in the macaque cortex. J Neurosci 32, 17465-17476

64 Young, M.P. (1992) Objective Analysis of the Topological Organization of the Primate Cortical Visual-System. Nature 358, 152-155

65 Ercsey-Ravasz, M., et al. (2013) A Predictive Network Model of Cerebral Cortical Connectivity Based on a Distance Rule. Neuron 80, 184-197

66 Harriger, L., et al. (2012) Rich club organization of macaque cerebral cortex and its role in network communication. PloS one 7, e46497

67 Goulas, A., et al. (2014) Comparative analysis of the macroscale structural connectivity in the macaque and human brain. PLoS computational biology 10, e1003529

68 Hagmann, P., et al. (2008) Mapping the structural core of human cerebral cortex. PLoS biology $6, \mathrm{e} 159$

69 Iturria-Medina, Y., et al. (2008) Studying the human brain anatomical network via diffusionweighted MRI and Graph Theory. NeuroImage 40, 1064-1076

70 Zalesky, A., et al. (2010) Whole-brain anatomical networks: does the choice of nodes matter? NeuroImage 50, 970-983

71 Gong, G., et al. (2009) Mapping anatomical connectivity patterns of human cerebral cortex using in vivo diffusion tensor imaging tractography. Cereb Cortex 19, 524-536

72 van den Heuvel, M.P., et al. (2010) Aberrant frontal and temporal complex network structure in schizophrenia: a graph theoretical analysis. J Neurosci 30, 15915-15926

73 Jones, D.K. (2010) Challenges and limitations of quantifying brain connectivity in vivo with diffusion MRI. Imaging Medicine 2, 14

74 Jbabdi, S. and Johansen-Berg, H. (2011) Tractography: where do we go from here? Brain connectivity 1, 169-183

75 Thomas, C., et al. (2014) Anatomical accuracy of brain connections derived from diffusion MRI tractography is inherently limited. Proceedings of the National Academy of Sciences of the United States of America 111, 16574-16579

76 van den Heuvel, M.P., et al. (2015) Comparison of diffusion tractography and tract-tracing measures of connectivity strength in rhesus macaque connectome. Human brain mapping 36, 30643075

77 Fornito, A., et al. (2013) Graph analysis of the human connectome: promise, progress, and pitfalls. NeuroImage 80, 426-444 
78 de Reus, M.A. and van den Heuvel, M.P. (2013) The parcellation-based connectome: Limitations and extensions. NeuroImage 80, 397-404

79 Van Essen, D.C., et al. (2012) The Human Connectome Project: a data acquisition perspective. NeuroImage 62, 2222-2231

80 Sporns, O. and Betzel, R.F. (2015) Modular Brain Networks. Annual review of psychology 81 Smith, S.M., et al. (2009) Correspondence of the brain's functional architecture during activation and rest. Proceedings of the National Academy of Sciences of the United States of America 106, 13040-13045

82 Greicius, M.D., et al. (2009) Resting-state functional connectivity reflects structural connectivity in the default mode network. Cereb Cortex 19, 72-78

83 Honey, C.J., et al. (2009) Predicting human resting-state functional connectivity from structural connectivity. Proceedings of the National Academy of Sciences of the United States of America 106, 2035-2040

84 van den Heuvel, M., et al. (2008) Microstructural organization of the cingulum tract and the level of default mode functional connectivity. J Neurosci 28, 10844-10851

85 van den Heuvel, M.P., et al. (2009) Functionally linked resting-state networks reflect the underlying structural connectivity architecture of the human brain. Human brain mapping 30, 31273141

86 Nijhuis, E.H., et al. (2013) Topographic hub maps of the human structural neocortical network. PloS one 8, e65511

87 van den Heuvel, M.P. and Sporns, O. (2013) Network hubs in the human brain. Trends in cognitive sciences $17,683-696$

88 Hinne, M., et al. (2015) Probabilistic clustering of the human connectome identifies communities and hubs. PloS one 10, e 0117179

89 van den Heuvel, M.P., et al. (2012) High-cost, high-capacity backbone for global brain communication. Proceedings of the National Academy of Sciences of the United States of America $109,11372-11377$

90 Collin, G., et al. (2014) Structural and Functional Aspects Relating to Cost and Benefit of Rich Club Organization in the Human Cerebral Cortex. Cerebral Cortex 24, 2258-2267

91 Grayson, D.S., et al. (2014) Structural and Functional Rich Club Organization of the Brain in Children and Adults. PloS one 9

92 Crossley, N.A., et al. (2014) The hubs of the human connectome are generally implicated in the anatomy of brain disorders. Brain 137, 2382-2395

93 Passingham, R.E., et al. (2002) The anatomical basis of functional localization in the cortex.

Nature reviews 3, 606-616

94 Schuz, A. (2002) Cortical Areas: Unity and Diversity. Taylor \& Francis

95 Fodor, J.A. (1983) Modularity of Mind: An Essay on Faculty Pscyhology. MIT Press

96 Gozzi, A. and Schwarz, A.J. (2016) Large-scale functional connectivity networks in the rodent brain. NeuroImage 127, 496-509

97 Stafford, J.M., et al. (2014) Large-scale topology and the default mode network in the mouse connectome. Proceedings of the National Academy of Sciences of the United States of America 111, $18745-18750$

98 Hutchison, R.M. and Everling, S. (2012) Monkey in the middle: why non-human primates are needed to bridge the gap in resting-state investigations. Frontiers in neuroanatomy 6, 29

99 Lu, H.B., et al. (2012) Rat brains also have a default mode network. Proceedings of the National Academy of Sciences of the United States of America 109, 3979-3984

100 Markov, N.T., et al. (2014) A Weighted and Directed Interareal Connectivity Matrix for Macaque Cerebral Cortex. Cerebral Cortex 24, 17-36

101 Betzel, R.F., et al. (2016) Generative models of the human connectome. NeuroImage 124, 1054-1064

102 Vertes, P.E., et al. (2012) Simple models of human brain functional networks. Proceedings of the National Academy of Sciences of the United States of America 109, 5868-5873 
103 Preuss, T.M. (2011) The human brain: rewired and running hot. Ann Ny Acad Sci 1225, E182E191

104 Rilling, J.K. (2014) Comparative primate neuroimaging: insights into human brain evolution. Trends in cognitive sciences 18, 46-55

105 Rilling, J.K. and Insel, T.R. (1999) Differential expansion of neural projection systems in primate brain evolution. Neuroreport 10, 1453-1459

106 Hopkins, W.D. and Rilling, J.K. (2000) A comparative MRI study of the relationship between neuroanatomical asymmetry and interhemispheric connectivity in primates: Implication for the evolution of functional asymmetries. Behav Neurosci 114, 739-748

107 Krubitzer, L.A. and Seelke, A.M.H. (2012) Cortical evolution in mammals: The bane and beauty of phenotypic variability. Proceedings of the National Academy of Sciences of the United States of America 109, 10647-10654

108 Barett, H.C. (2013) A Hierarchical Model of the Evolution of Human Brain Specializations. In In the Light of Evolution:

Volume VI: Brain and Behavior (2013) (Striedter, G.F., et al., eds), National Academy of Sciences 109 Krubitzer, L. (2007) The magnificent compromise: cortical field evolution in mammals.

Neuron 56, 201-208

110 Rilling, J.K., et al. (2008) The evolution of the arcuate fasciculus revealed with comparative DTI. Nature neuroscience 11, 426-428

111 Gotts, S.J., et al. (2013) Two distinct forms of functional lateralization in the human brain. Proceedings of the National Academy of Sciences of the United States of America 110, E3435-3444 112 Hopkins, W.D., et al. (2007) The association between handedness, brain asymmetries, and corpus callosum size in chimpanzees (Pan troglodytes). Cerebral Cortex 17, 1757-1765

113 Nicosia, V., et al. (2013) Phase transition in the economically modeled growth of a cellular nervous system. Proceedings of the National Academy of Sciences of the United States of America $110,7880-7885$

114 van den Heuvel, M.P. and Sporns, O. (2011) Rich-club organization of the human connectome. J Neurosci 31, 15775-15786

115 Nigam, S., et al. (2016) Rich-Club Organization in Effective Connectivity among Cortical Neurons. Journal of Neuroscience 36, 670-684

116 Schroeter, M.S., et al. (2015) Emergence of Rich-Club Topology and Coordinated Dynamics in Development of Hippocampal Functional Networks In Vitro. Journal of Neuroscience 35, 54595470

117 Binicewicz, F.Z., et al. (2015) Graph analysis of the anatomical network organization of the hippocampal formation and parahippocampal region in the rat. Brain structure \& function Epub ahead of print

118 Senden, M., et al. (2014) Rich club organization supports a diverse set of functional network configurations. Neurolmage 96, 174-182

119 de Reus, M.A. and van den Heuvel, M.P. (2014) Simulated rich club lesioning in brain networks: a scaffold for communication and integration? Frontiers in human neuroscience 8, 647 120 Baggio, H.C., et al. (2015) Rich Club Organization and Cognitive Performance in Healthy Older Participants. Journal of cognitive neuroscience 27, 1801-1810

121 Dehaene, S., et al. (1998) A neuronal model of a global workspace in effortful cognitive tasks. Proceedings of the National Academy of Sciences of the United States of America 95, 14529-14534 122 Dehaene, S. and Naccache, L. (2001) Towards a cognitive neuroscience of consciousness: basic evidence and a workspace framework. Cognition 79, 1-37

123 Kitzbichler, M.G., et al. (2011) Cognitive Effort Drives Workspace Configuration of Human Brain Functional Networks. Journal of Neuroscience 31, 8259-8270

124 Shanahan, M. (2012) The brain's connective core and its role in animal cognition.

Philosophical transactions of the Royal Society of London. Series B, Biological sciences 367, 27042714 
125 Bassett, D.S., et al. (2008) Hierarchical organization of human cortical networks in health and schizophrenia. $J$ Neurosci 28, 9239-9248

126 Mesulam, M.M. (1998) From sensation to cognition. Brain 121 ( Pt 6), 1013-1052

127 Wylie, K.P., et al. (2015) Between-network connectivity occurs in brain regions lacking layer IV input. NeuroImage 116, 50-58

128 van den Heuvel, M.P., et al. (2015) Associated Microscale Spine Density and Macroscale

Connectivity Disruptions in Schizophrenia. Biological psychiatry

129 van den Heuvel, M.P., et al. (2015) Bridging Cytoarchitectonics and Connectomics in Human

Cerebral Cortex. J Neurosci 35, 13943-13948

130 Beul, S.F., et al. (2015) A predictive model of the cat cortical connectome based on cytoarchitecture and distance. Brain structure \& function 220, 3167-3184

131 Elston, G.N. (2003) Cortex, cognition and the cell: new insights into the pyramidal neuron and prefrontal function. Cereb Cortex 13, 1124-1138

132 Tomasi, D., et al. (2013) Energetic cost of brain functional connectivity. Proceedings of the National Academy of Sciences of the United States of America 110, 13642-13647

133 Power, J.D., et al. (2011) Functional network organization of the human brain. Neuron 72, 665678

134 van den Heuvel, M.P. and Sporns, O. (2013) An anatomical infrastructure for integration between functional networks in human cerebral cortex. $J$ Neurosci 33, 11

135 Crossley, N.A., et al. (2013) Cognitive relevance of the community structure of the human brain functional coactivation network. Proceedings of the National Academy of Sciences of the United States of America 110, 11583-11588

136 Samu, D., et al. (2014) Influence of wiring cost on the large-scale architecture of human cortical connectivity. PLoS computational biology 10, e1003557

137 Roberts, J.A., et al. (2016) The contribution of geometry to the human connectome.

NeuroImage 124, 379-393

138 Budd, J.M. and Kisvarday, Z.F. (2012) Communication and wiring in the cortical connectome. Frontiers in neuroanatomy 6, 42

139 Sammut, M., et al. (2015) Glia-derived neurons are required for sex-specific learning in C. elegans. Nature 526, 385-390

140 Rilling, J.K., et al. (2012) Differences between chimpanzees and bonobos in neural systems supporting social cognition. Social cognitive and affective neuroscience 7, 369-379

141 Parish, A.R. and De Waal, F.B. (2000) The other "closest living relative". How bonobos (Pan paniscus) challenge traditional assumptions about females, dominance, intra- and intersexual interactions, and hominid evolution. Annals of the New York Academy of Sciences 907, 97-113 142 Bickart, K.C., et al. (2011) Amygdala volume and social network size in humans. Nature neuroscience 14, 163-164

143 Adolphs, R. (2010) What does the amygdala contribute to social cognition? Year in Cognitive Neuroscience 2010 1191, 42-61

144 Friederici, A.D. (2009) Pathways to language: fiber tracts in the human brain. Trends in cognitive sciences $13,175-181$

$145 \mathrm{Li}, \mathrm{L}$. , et al. (2013) Mapping putative hubs in human, chimpanzee and rhesus macaque connectomes via diffusion tractography. NeuroImage 80, 462-474

146 Miranda-Dominguez, O., et al. (2014) Bridging the gap between the human and macaque connectome: a quantitative comparison of global interspecies structure-function relationships and network topology. J Neurosci 34, 5552-5563

147 Bruner, E., et al. (2016) Evidence for expansion of the precuneus in human evolution. Brain structure \& function

148 Zalesky, A., et al. (2011) Disrupted axonal fiber connectivity in schizophrenia. Biological psychiatry 69, 80-89

149 Ryman, S.G., et al. (2014) Sex differences in the relationship between white matter connectivity and creativity. NeuroImage 101, 380-389 
150 Li, Y., et al. (2009) Brain anatomical network and intelligence. PLoS computational biology 5, e1000395

151 van den Heuvel, M.P., et al. (2009) Efficiency of functional brain networks and intellectual performance. J Neurosci 29, 7619-7624

152 Giessing, C., et al. (2013) Human Brain Functional Network Changes Associated with

Enhanced and Impaired Attentional Task Performance. Journal of Neuroscience 33, 5903-5914

153 Deco, G., et al. (2015) Rethinking segregation and integration: contributions of whole-brain modelling. Nature reviews 16, 430-439

154 Zamora-Lopez, G., et al. (2010) Cortical hubs form a module for multisensory integration on top of the hierarchy of cortical networks. Frontiers in neuroinformatics 4, 1

155 Schmidt, R., et al. (2015) Kuramoto model simulation of neural hubs and dynamic synchrony in the human cerebral connectome. BMC neuroscience 16, 54

156 Swanson, L.W. (2011) Brain Architecture. Oxford University Press

157 Avena-Koenigsberger, A., et al. (2015) Network morphospace. J R Soc Interface 12

158 Roth, G. and Dicke, U. (2005) Evolution of the brain and intelligence. Trends in cognitive sciences $9,250-257$

159 Fox, D. (2011) The Limits of Intelligence. Scientific American 37

160 Krubitzer, L. and Stolzenberg, D.S. (2014) The evolutionary masquerade: genetic and epigenetic contributions to the neocortex. Current opinion in neurobiology 24, 157-165

161 Chung, K. and Deisseroth, K. (2013) CLARITY for mapping the nervous system. Nat Methods $10,508-513$

162 Reid, A.T., et al. (2016) A cross-modal, cross-species comparison of connectivity measures in the primate brain. NeuroImage 125, 311-331

163 Azadbakht, H., et al. (2015) Validation of High-Resolution Tractography Against In Vivo

Tracing in the Macaque Visual Cortex. Cereb Cortex 25, 4299-4309

164 Katsuki, T. and Greenspan, R.J. (2013) Jellyfish nervous systems. Current Biology 23, R592R594

165 Satterlie, R.A. (2011) Do jellyfish have central nervous systems? J Exp Biol 214, 1215-1223

166 Garm, A., et al. (2007) The ring nerve of the box jellyfish Tripedalia cystophora. Cell Tissue Res 329, 147-157

167 Dunne, J.F., et al. (1985) A subset of cells in the nerve net of Hydra oligactis defined by a monoclonal antibody: its arrangement and development. Developmental biology 109, 41-53

168 Garm, A., et al. (2006) Rhopalia are integrated parts of the central nervous system in box jellyfish. Cell Tissue Res 325, 333-343

169 Koizumi, O., et al. (2015) The nerve ring in cnidarians: its presence and structure in hydrozoan medusae. Zoology 118, 79-88

170 Colizza, V., et al. (2006) Detecting rich-club ordering in complex networks. Nature Physics 2, 110-115

171 Goni, J., et al. (2013) Exploring the Morphospace of Communication Efficiency in Complex Networks. PloS one 8

172 Banerjee, A. and Jost, J. (2009) Graph spectra as a systematic tool in computational biology.

Discrete Appl Math 157, 2425-2431

173 de Lange, S.C., et al. (2014) The Laplacian spectrum of neural networks. Frontiers in computational neuroscience 7, 189

174 Sole, R. and Valverde, S. (2004) Information Theory of Complex Networks: On Evolution and Architec- tural Constraints. Lect. Notes Phys. 650, 18

175 Scholtens, L.H., et al. (2014) Linking macroscale graph analytical organization to microscale neuroarchitectonics in the macaque connectome. J Neurosci 34, 12192-12205 


\section{connectomes}
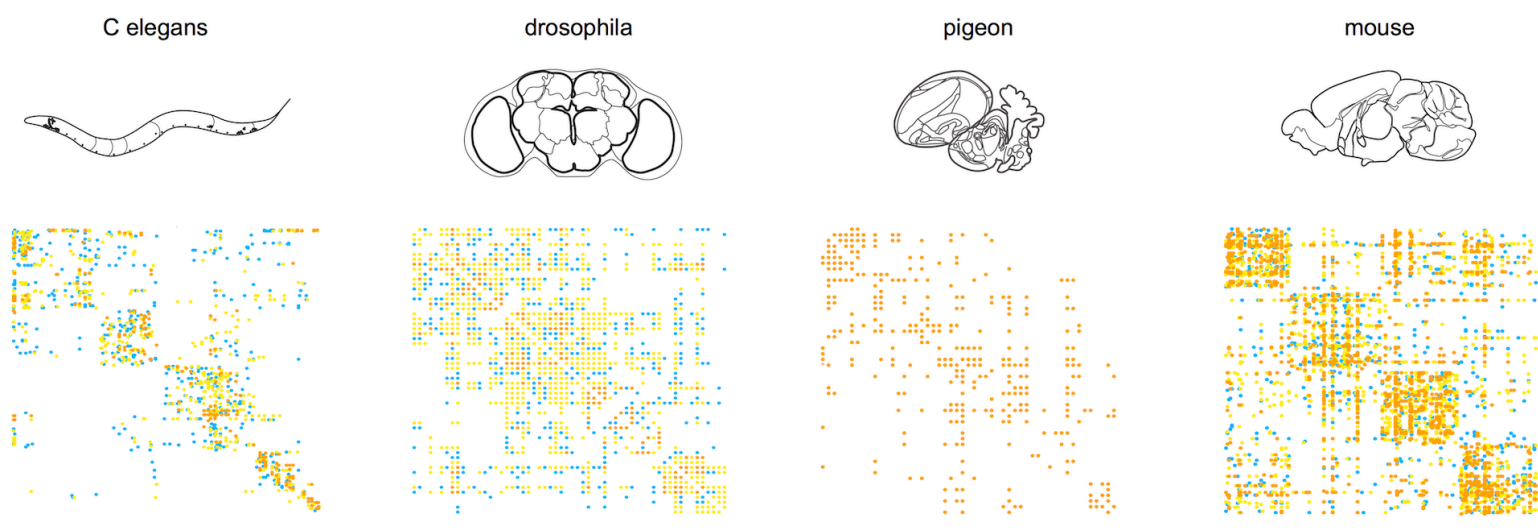

rat

cat
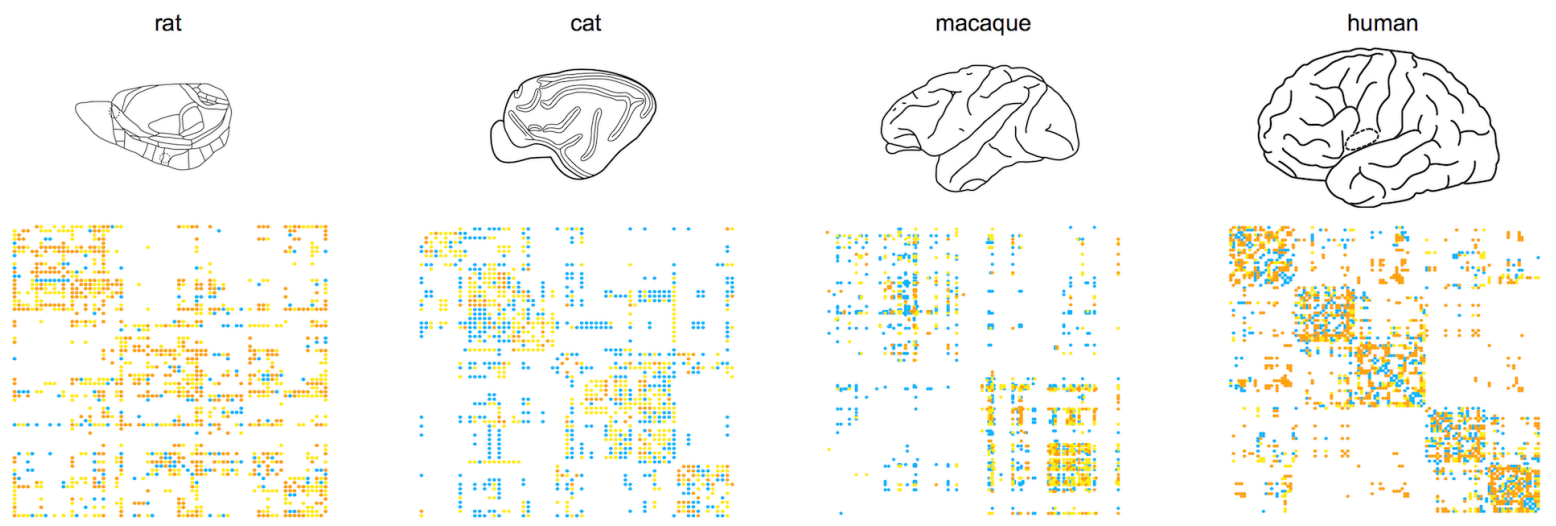


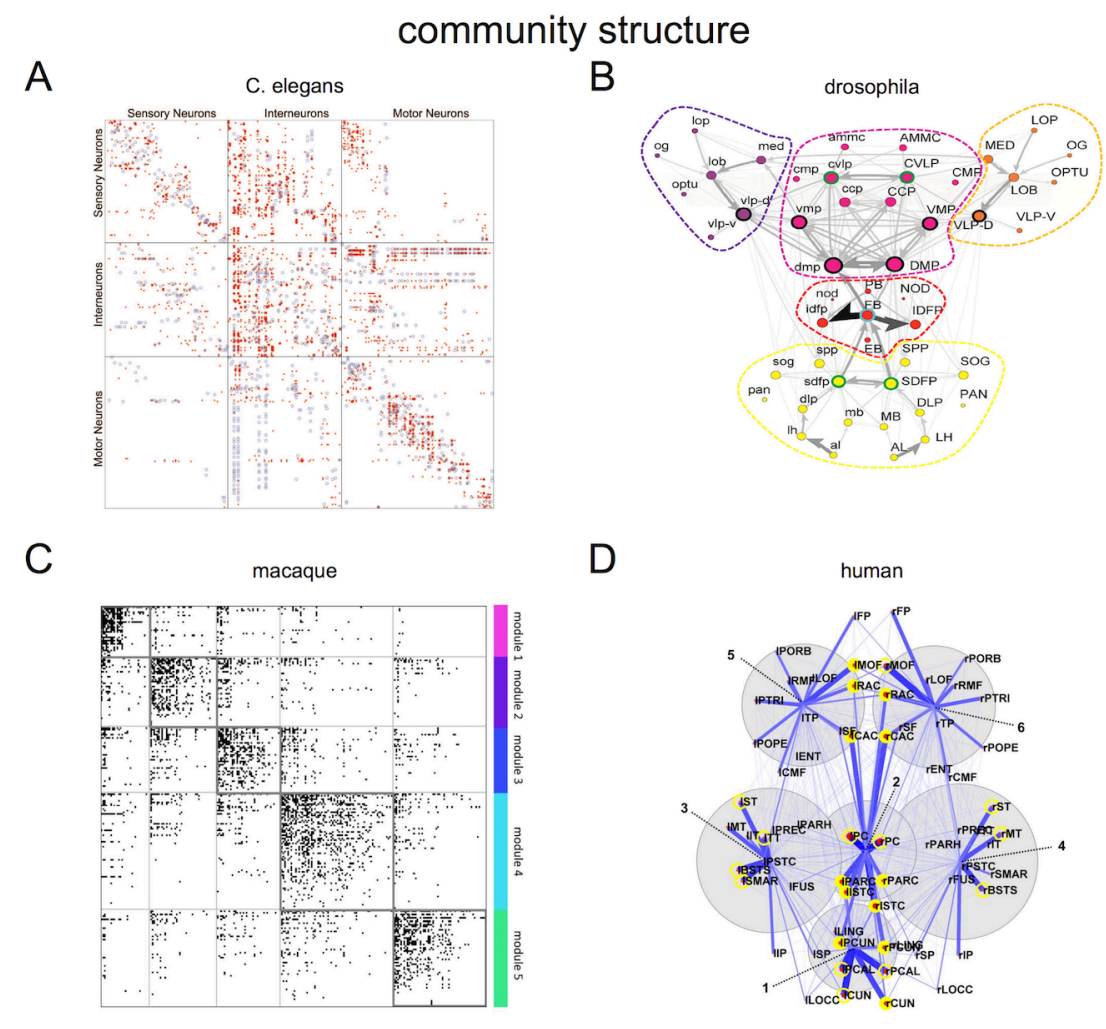


hubs and rich club organization

A

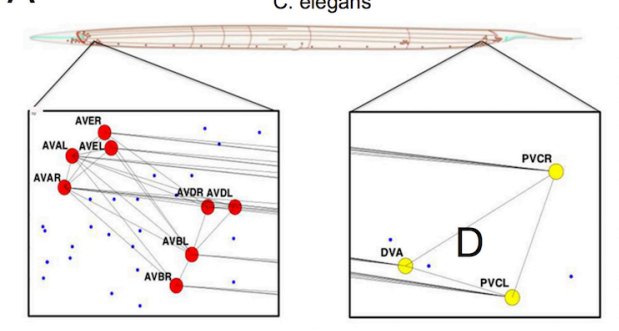

C

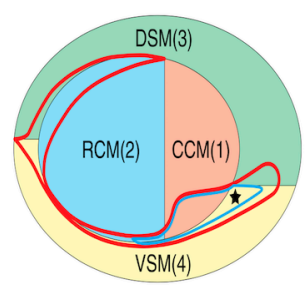

B

mouse Brainstem-
Cerebellum

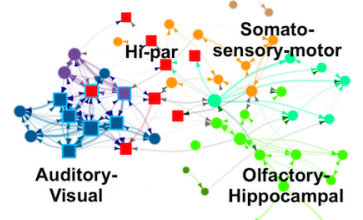

E

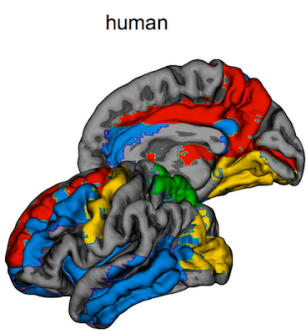




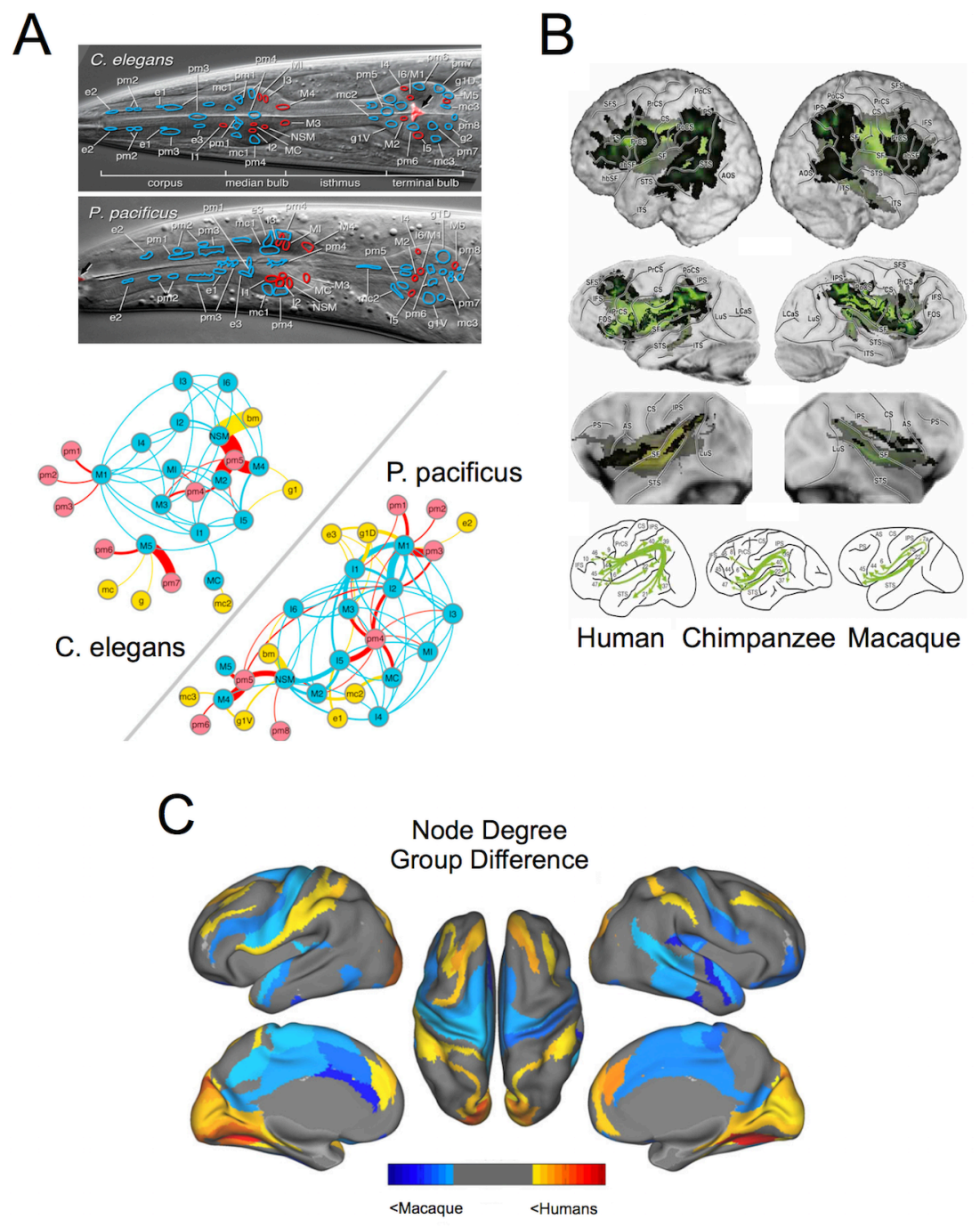




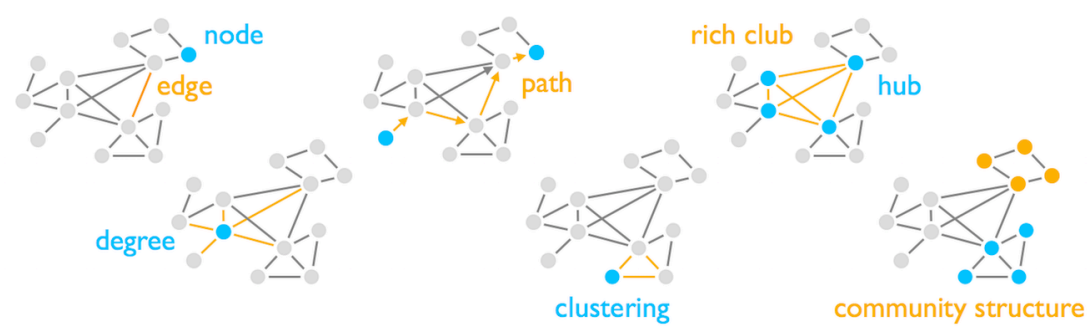




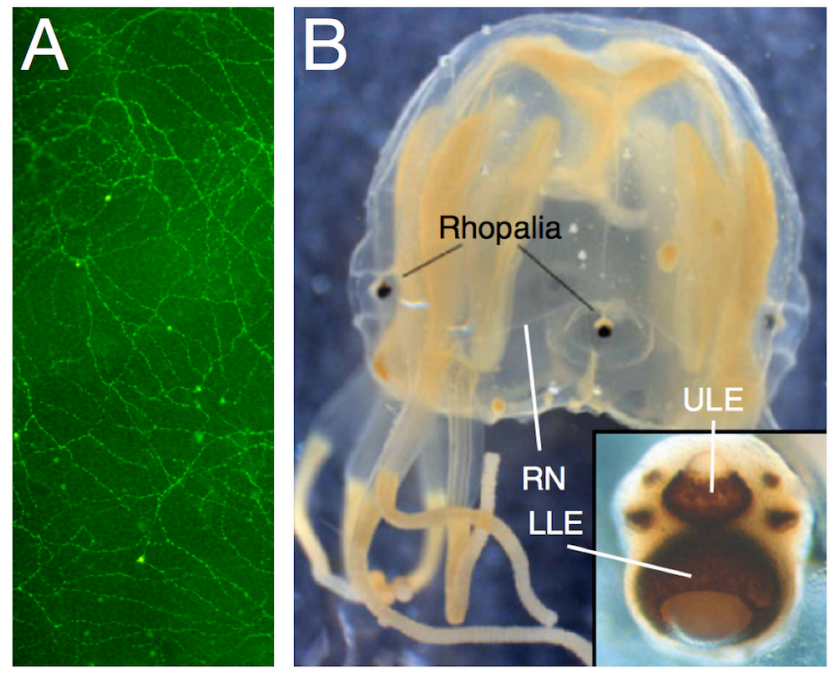

